\title{
Einleitung \\ Zukunftsbilanz. Annäherungen an transnationale Feminismen 25 Jahre nach Peking
}

Eine Zukunftsbilanz zu schreiben - das mag grundsätzlich nach einem unmöglichen, zumindest aber nach einem widersprüchlichen Vorhaben klingen. Bilanzen rechnen bekanntlich Vorhandenes und damit in aller Regel Vergangenes ab. Dagegen will das vorliegende Heft zum historischen Datum der im September 2020 nunmehr 25 Jahre zurückliegenden Weltfrauenkonferenz von Peking den Blick nach vorne richten und aus der Gegenwart heraus auch etwas über zukünftige Erfordernisse aussagen. Zukunftsbilanz scheint uns ein guter Begriff zu sein, sich den vielfältigen und in vielfacher Hinsicht differenten Gegenwarten transnationaler Feminismen zu nähern, indem wir sie vor dem Hintergrund der Geschichte transnationaler Geschlechterpolitiken (auch) als Verweise auf die Zukunft verstehen. Anders formuliert besteht unser Anliegen nicht vordringlich darin, den umfangreichen, zu den vorherigen Jubiläumsdaten der Pekinger Konferenz erschienenen Bilanzen transnationaler Feminismen (im Kontext von Peking plus 10 z. B. Moghadam 2005; zu Peking plus 20 z. B. Baksh / Harcourt 2015) eine weitere Version hinzuzufügen, die ebenfalls die Linien von Peking in die Gegenwart nachverfolgt.

Ganz zweifellos prägen bei aller Wechselhaftigkeit internationaler Konfliktsituationen wesentliche Kontinuitäten die Weltpolitik und die meisten der großen Fragen transnationaler Geschlechterpolitik stellen sich heute ebenso wie vor 25 Jahren. Die Zusammenhänge von globalem Kapitalismus, asymmetrischen Nord-Süd-Verhältnissen, ökologischer Verheerung, ökonomischen und sozialen Ungleichheitslagen und Verletzungen der Menschenrechte bilden nach wie vor den einen der beiden zentralen Komplexe feministischer Kämpfe um sgute und nachhaltige Weltentwicklung(en). Der andere geht hervor aus den Aus- und Verhandlungen von Staatlichkeit, Staatsbürgerschaft, Nation und Nationalismen, Religionen und Fundamentalismen, Militarismus bzw. Friedenspolitiken und Konfliktlösung. Dazwischen und quer dazu liegt das weite Feld der Körperpolitiken, in dem immer noch um Selbstbestimmung und Identitäten gerungen und zugleich die verschiedensten Formen vergeschlechtlichter und sexualisierter Gewalt bekämpft werden. 
Gleichzeitig hat sich die Welt hinsichtlich all dieser Kontexte in den letzten Jahrzehnten rasant verändert, insbesondere mit Blick auf die Beschleunigung von Globalisierung und Finanzialisierung, die Ausmaße von wiederkehrenden ökonomischen Krisen, ökologischer Zerstörung und Nahrungsmittelkrisen und die Zunahme von Militarismen, Autoritarismen, Rassismen und Fundamentalismen im 21. Jahrhundert.

Aus Geschlechterperspektive haben sich grundlegende Strukturen von Ungleichheit, Ausbeutung und Unterdrückung, insbesondere entlang der Gender Kategorie(n) in diesen Prozessen als nahezu unerschütterlich erwiesen und sich in vielen Bereichen sogar vertieft und verstärkt. Wir leben heute in einer Welt, in der es mehr Wohlstand aber zugleich auch mehr und größere Ungleichheit gibt als zu irgendeinem anderen Zeitpunkt seit dem Ende des Zweiten Weltkriegs (vgl. UN Women 2016, 3 ff.) Dies impliziert einerseits Unterschiede zwischen Frauen ${ }^{\star}$, die so groß sind wie nie zuvor, und andererseits, dass Frauen ${ }^{\star}$ von sämtlichen Formen der Ungleichheit, Marginalisierung, Diskriminierung und Gewalt stärker betroffen sind als Männer ${ }^{\star}$. Trotz jahrzehntelanger Prozesse transnationaler feministischer Selbstorganisation und Vernetzung, trotz Lobbying und Gender Mainstreaming und trotz feministischer Partizipation in allen Bereichen transnationaler Gesellschaftspolitik sind die wesentlichen Strukturen und Prozesse der internationalen Wirtschafts- und Handelspolitik ebenso wie die der Militär- und Sicherheitspolitik weitgehend persistent und resistent gegen feministische Interventionen geblieben.

Doch so gewiss wie bestimmte Grundmuster von Ungleichheit die verschiedenen Phasen von Weltentwicklung der letzten Jahrzehnte überdauert haben, so verändern sich mit den Verschiebungen innerhalb und zwischen den Grundstrukturen der Weltpolitik auch die transnationalen feministischen Diskurse der Kritik, die Strategien der politischen Herausforderung und die gegenhegemonialen sozialen und kulturellen Praxen. Diese feministischen Transformationen und die daraus hervorgehenden Dynamiken, die Veränderungen transnationaler feministischer Diskurse,

1 Im Prozess der Weltfrauenkonferenz in Peking spielten die für die meisten Feminismen der Welt heute bedeutsamen Anstrengungen um die Dekonstruktion der Genderkategorien und insbesondere der binären Zweigeschlechtlichkeit noch kaum eine Rolle. Gleiches gilt - mehr oder weniger - für die verschiedenen Gender Ansätze, Gender 'machineries und Gender Forschungen im Kontext der Vereinten Nationen bis heute. Während langjährige feministische Interventionen schließlich erfolgreich dazu geführt haben, dass einige der Statistiken der UN geschlechtsdifferenziert ausgewiesen werden, beschränkt sich diese Differenzierung auf die herkömmlichen Kategorien der Zweigeschlechtlichkeit. Mit einer bewusst geführten ${ }_{-}$-Schreibweise soll dieses Defizit hier zumindest bezeichnet werden. 
Themen und Praxen wollen wir hier in den Fokus der Diskussion rücken und damit eine zukunftsbezogene Lesart von Entwicklungen transnationaler Feminismen von Peking zur Gegenwart vorschlagen.

\section{Vergangene Perspektiven}

Weltweite Sichtbarkeit, regionale und lokale Verankerungen transnationaler Bewegungen und Organisierungsprozesse, funktionierende Netzwerke, strategische Vielfalt, politisches Knowhow, Reaktionsschnelligkeit, thematische Breite und Expertise, argumentative Schärfe, Streitbarkeit und Reflektionstiefe nach innen - all diese Stärken und Kompetenzen transnationaler Feminismen, die in Peking weltöffentlich sichtbar wurden, hatten sich transnationale Frauenbewegungen, ihre Organisationen und Netzwerke zuvor über zwei Jahrzehnte hinweg erstritten und erarbeitet (vgl. exempl. Wichterich 1995, Lenz / Schwenken 2001, Antrobus 2004).

Während der Weltfrauendekade von 1975 bis 1985 und im Kontext der drei Weltfrauenkonferenzen 1975 in Mexiko City, 1980 in Kopenhagen und 1985 in Nairobi waren innerhalb der südlichen Kontinente tragfähige Strukturen regionaler feministischer Vernetzung gewachsen. Die Beiträge von Boyce Davies, Leinius und Mageza-Barthel in diesem Heft verweisen auf verschiedene Aspekte dieser Prozesse. Zahlreiche politische wie inhaltliche Konflikte und Widersprüche in und zwischen den Bewegungen weltweit, die auch die Grundstruktur der weltpolitischen Konfliktlagen Nord-Süd, Ost-West und Nahost widerspiegelten, wurden in dieser Zeit zum Gegenstand permanenter, heftiger Auseinandersetzungen. 1984 gründete sich das feministische Süd-Süd-Netzwerk DAWN (Development Alternatives with Women for a New Era), das bei der Konferenz in Nairobi zum ersten Mal und von da an kontinuierlich mit seinen scharfen Analysen und Konzeptionen die Hegemonie weißer, westlicher Feministinnen im transnationalen Feminismus massiv herausforderte. DAWNs "süd-feministische Kritiken ${ }^{2}$ von Weltentwicklung, Militarismus, Fundamentalismus und globalen Krisenphänomenen wie wiederkehrende Ernährungs- und Schuldenkrisen hatten großen Einfluss auf die Debatten in Nairobi und bei allen nachfolgenden Weltkonferenzen. Für kritische feministische Diskurse über Weltentwicklung, transnationale Gerechtigkeit und transnationalen Feminismus waren die Beiträge des Netzwerkes

2 Vgl. zu dieser Selbstbeschreibung, zur Geschichte und Arbeit von DAWN deren Netzauftritt unter https://dawnnet.org/ 
und waren feministische Perspektiven des Globalen Südens fortan grundlegend (exempl. Grown/Sen 1987; Reichmann / Correa 1994; Sen / Durano 2015).

In der ersten Hälfte der 1990er Jahre eröffnete dann die Serie der Weltkonferenzen der Vereinten Nationen, die nach dem Ende des Ost-WestKonflikts die entscheidenden Probleme globaler Entwicklung weltöffentlich verhandelte, der transnationalen Geschlechterpolitik ganz neue Chancen der Partizipation an den Prozessen und Institutionen transnationaler Gesellschaftspolitik. 1992 beim Weltumweltgipfel in Rio de Janeiro konnten sich Genderperspektiven erstmals in der Geschichte der Vereinten Nationen als ein Querschnittsthema der Diskussionen um Weltentwicklung (und Nachhaltigkeit) etablieren; 1993 beim Menschenrechtsgipfel in Wien wurden grundlegende Erfolge für die Durchsetzung von FrauenMenschenrechten weltweit errungen; 1994 bei der Weltbevölkerungskonferenz in Kairo wurden feministische Ansprüche und Definitionen reproduktiver Rechte von Frauen zum Gegenstand der Weltpolitik. Auch diese Phase feministischer Politik in den sich neu formierenden Strukturen von 'Global Governancer ist sehr gut erforscht und die errungenen Verhandlungserfolge sind vielfach beschrieben (exempl. Meyer/Prügl 1999; HollandCunz/Ruppert 2000). Sie bereiteten den Boden für den endgültigen Durchbruch in der Etablierung von Gender Mainstreaming als legitimer Anspruch an die Institutionen und Verfahren der Weltpolitik bei der Pekinger Weltfrauenkonferenz 1995.

Vor allem in den ersten Jahren nach der Konferenz übersetzten Akteur^innen der Geschlechterpolitik weltweit die erreichte Sichtbarkeit und die politischen Stärken transnationaler feministischer Selbstorganisierung auf allen Ebenen des politischen Handelns in Antidiskriminierungs- und Gleichheitspolitiken. In vielen Nationalstaaten, in regionalen wie in internationalen Institutionen wurden sogenannte gender machineries etabliert, Gesetze und Maßnahmen gegen sexualisierte Gewalt durchgesetzt, Antidiskriminierungsgesetze verabschiedet, Quoten für die politische Repräsentation von Frauen eingeführt und gendersensitive Policy-Planungen, vor allem in den Politikfeldern Gesundheit und Bildung, entwickelt. Rechtsreformen wurden eingeleitet und umgesetzt, die von Änderungen im Zivilrecht über die Neuformulierung von Verfassungen bis zum Fakultativprotokoll zur Frauenrechtskonvention CEDAW reichten (vgl. United Nations 2015). Nicht zuletzt mit diesen Kodifizierungen von Gleichheitsansprüchen, die zweifellos zu den wesentlichen Grundlagen von Geschlechtergerechtigkeit zählen, schienen sich feministische Hoff- 
nungen auf die Durchsetzung emanzipatorischer Forderungen im Kontext von Global Governance teilweise zu realisieren.

Dabei waren diese Erfolge aber stets ambivalent, da sie als ihre Kehrseite immer auch innerfeministisch heftig umstrittene Politikaspekte beinhalteten. Je nach Anschlussfähigkeit der betreffenden Policy-Felder variieren die Erfolgsaussichten feministischer Politik (vgl. Caglar et al. 2013). Oft genug besteht der Eintrittspreis zu Institutionen und Verhandlungsrunden der internationalen Politik in der vorauseilenden Entradikalisierung feministischer Ansätze, der Anpassung an liberale Paradigmen institutioneller

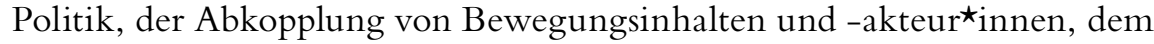
kompletten Ausschluss subalterner Positionen und nicht zuletzt der Reproduktion von Topoi oder sogar Stereotypen wie etwa Frauen als smost vulnerable group , die eigentlich im Zentrum feministischer Kritik stehen.

Besonders markant lassen sich diese Ambivalenzen und Widersprüche an der Politik der FrauenMenschenrechte aufzeigen, die seit den 1990er Jahren die vielleicht entscheidendsten Erfolge transnationaler feministischer Politik errungen hat. Seitdem es 1993 in Wien gelungen war, das internationale Menschenrechtsregime auf den Schutz vor sexualisierter und vergeschlechtlichter Gewalt zu verpflichten, ist die Politik der FrauenMenschenrechte ein zentraler Gegenstand der innerfeministischen Kontroverse. Zum einen durchzieht der feministische sSüd-Nord-Konflikt die transnationale FrauenMenschenrechtspolitik auf besondere Weise. Seit Beginn der antikolonialen Kämpfe in den südlichen Ländern, an denen Frauenbewegungen beinahe überall maßgeblich beteiligt waren und die sich parallel zu den Befreiungskämpfen mit Frauenbewegungen anderer Länder und Regionen zusammenschlossen, um gemeinsam für Geschlechtergleichheit einzutreten (vgl. die Beiträge von Boyce Davies und Mageza-Barthel), standen Konflikte um Fragen sozialer Gleichheit und Gerechtigkeit im Mittelpunkt der Auseinandersetzung zwischen Süd und Nord. Während Frauenbewegungen im Süden gegen die internationale Machtordnung, gegen Imperialismus, koloniale Ausbeutung und Unterdrückung kämpften, traten Frauenbewegungen im Norden mehrheitlich für Fragen der politischen Gleichheit und für Partizipationsrechte ein ${ }^{3}$. Bis heute verlaufen bewegungsinterne Auseinandersetzungen um die Implementierung von FrauenMenschenrechten im UN-Kontext entlang die-

3 Von den Bewegungen Schwarzer Frauen wurde die hegemoniale Mehrheitsweiße Gleichstellungsperspektive überall im Norden bereits in den späten 1970er Jahren massiv herausgefordert (exempl. Combahee River Collective 1978). In den 1990er Jahren verbanden sich ihre Forderungen vielfach mit denen von Migrant $\star_{i n n e n}$ (exempl. Uremović / Oerter 1994) 
ser Konfliktlinie. Faktisch besteht kein Zweifel daran, dass entgegen aller feministischen Bekenntnisse zur Unteilbarkeit der beiden Generationen politischer und sozialer Menschenrechte der Schwerpunkt der konkreten Umsetzungen von FrauenMenschenrechten stets auf Teilhaberechten liegt (vgl. Zwingel 2012, 118-120). Schon frühzeitig haben kritische feministische Stimmen aus dem globalen Süden dieses Ungleichgewicht damit in Verbindung gebracht, dass die Begrenzung der Reichweite von FrauenMenschenrechten bereits in ihrem Ursprung im westlich-liberalen Konzept von Staatlichkeit und Menschenrechten angelegt ist (vgl. Schild 2014). Entsprechend haben Feminist ${ }^{\star}$ innen aus dem Osten Europas und aus den südlichen Kontinenten immer wieder die Überlagerung systemkritischer feministischer Politikansätze durch die internationale 'women's human rights machinery beanstandet. Insbesondere die finanziellen Maßgaben der FrauenMenschenrechte-Förderkonjunktur sorg(t)en nach Ansicht feministischer Kritiker`innen für die einseitige Ausrichtung der Politik von geberabhängigen Frauenorganisationen (Ruppert 2015, 113).

Eine zweite Dimension der Kritik betrifft die Funktionalisierbarkeit und Vereinnahmbarkeit des Ansatzes. Spätestens mit der US-geführten Invasion in Afghanistan 2001, die international nicht zuletzt mit dem Kampf gegen die Unterdrückung von Frauen durch die Taliban legitimiert worden war, rückte die Instrumentalisierbarkeit des FrauenMenschenrechtsansatzes für hegemoniale Interessen stärker ins Zentrum feministischer Auseinandersetzungen. Auch wenn die Rechtfertigung von Krieg mit der Befreiung der Frauen der "Anderen" historisch alles andere als neu ist und die Kontinuität kolonialer Wissensbestände bzw. neokoloniale Ausformung vorherrschender Rechtsdiskurse (McLaren 2017, 83) schon lange zuvor aus der Perspektive feministischer postcolonial studies angegriffen wurden, markierte der Afghanistan Krieg einen tiefen Einschnitt in den Auseinandersetzungen über die Ein- und Ausschlüsse transnationaler Frauen- und Geschlechterpolitiken (vgl. Kapur 2002).

Mittelfristig, so lässt sich in der Rückschau heute resümieren, hat sich das "Peking-Momentum" transnationaler Frauenbewegungs- und Geschlechterpolitik gewissermaßen selbst verbraucht. Neue Politikchancen, die mit der breiten Mobilisierung, der Pluralisierung und Professionalisierung der Bewegungen entstanden waren, wurden von den Institutionen, in die sie eingebracht wurden, im Laufe der Jahre weitgehend aufgesogen oder inkorporiert. Politische Konflikte und epistemologische Differenzen in und zwischen den verschiedenen feministischen Diskursen und Ansätze ihrer politischen und analytischen Bearbeitung traten (erneut) in den Vordergrund; Verhältnisse zwischen den lokalen, regionalen und inter- 
nationalen Dimensionen des Transnationalen wurden (wieder) verstärkt verhandelt und Süd-Süd-Diskurse gewannen an Dynamik und Einfluss. Insofern betraf die erhoffte 'Transformation durch Partizipation« letztlich mindestens ebenso die Strukturen und Praxen transnationaler Feminismen selbst wie die Institutionen und Kontexte internationaler Politik, auf die sie zielten.

\section{Transnationale Diskurse}

Die Pekingkonferenz stellte somit nicht nur hinsichtlich der Politikchancen feministischer Akteur $\star_{i n n e n}$ einen Wendepunkt dar. Fortan veränderten sich auch bestimmte normative und theoretische Vorstellungen von transnationalem Feminismus, insbesondere bezüglich der Raum-Zeit-Dimensionen, innerhalb derer sich transnationale feministische Praxen verorten und der politischen Konsequenzen, die damit einhergehen. Prominent wurde das Trans im grenzüberschreitenden Feminismus und damit die Ideen, Begriffe und Konzepte, die transnationale Feminismen bestimmen, überhaupt erst nach Peking. Bis 1995 war auch dann, wenn Bewegungs- und Zivilgesellschaftspolitiken den Gegenstand der Diskussion bildeten, eigentlich kaum von transnationalem Feminismus die Rede gewesen, sondern in der Tradition linker, internationalistischer Politik oder in akademischen Perspektiven aus dem Umfeld der Internationalen Beziehungsforschung von "internationaler Frauenpolitik" oder »internationaler Geschlechterpolitik" (exempl. Ruppert 1998, Braig / Wölte 2003).

Im deutschsprachigen Raum gab es nur sehr wenige und nicht allzu erfolgreiche Versuche, die Politik feministischer Netzwerke systematisch von der Politik der Staaten und deren sinter-nationalen Institutionen zu unterscheiden (vgl. Ruppert 2001) und erst mit dem Aufstieg des Transnationalismus Paradigmas in der Migrationsforschung im neuen Millennium (vgl. Lutz/Amelina 2017) setzte sich hierzulande die begriffliche Wendung zum transnationalen Feminismus tatsächlich durch.

Dabei waren in der anglo-amerikanischen Debatte schon einige Jahre zuvor mehrere theoretisch klar voneinander zu unterscheidende, für den wissenschaftlichen wie den politischen Feminismus sehr bedeutsame Beiträge über Konzepte, Inhalte und Bedeutungen transnationaler Feminismen in die Diskussion gebracht worden. Als grundlegend für die gesamte weitere Entwicklung von Theorien transnationaler Feminismen greifen wir hier insbesondere den aus poststrukturalistisch-postmoderner Perspektive argumentierenden Text von Inderpal Grewal und Caren Kaplan 
(1994) sowie einen zweiten, materialistisch-dekolonialen Text von Jacqui Alexander und Chandra Mohanty (1997) heraus. Beide Texte gelten als paradigmatisch für die weitere westlich-englischsprachige Diskussion über transnationale Feminismen (vgl. Desai 2015). Eine dritte, aus den internationalen Beziehungen und weniger aus dem Feminismus kommende, institutionalistisch-pluralistische Linie der Diskussion führen wir hier auf einen maßgeblichen Beitrag von Margaret Keck und Kathryn Sikkink (1998) zurück. Alle drei Ansätze reflektieren und theoretisieren bestimmte Ausschnitte der politischen Realitäten und Praxen transnationaler Feminismen.

Beide dekolonial-feministischen Texte sind systematisierende Einführungen zu Sammelbänden, die den doppelten Anspruch erheben, feministische Theoriebildung herauszufordernd voranzubringen und transnationale feministische Allianzen politisch zu stärken, indem sie "intersections" von (feministisch)postkolonialen und (feministisch)postmodernen Diskursen mit Ansätzen linker, feministischer Theorie und Praxis herausarbeiten. Das zentrale Anliegen von Grewal/Kaplan ist dabei die im postmodernen Sinne radikale Dezentrierung der gender Kategorie(n) durch die Dekonstruktion ihrer räumlichen, d.h. geographischen und geopolitischen Dimensionen. Grewal/Kaplan wenden sich entschieden gegen Dichotomisierungen wie lokal-global oder Zentrum - Peripherie und sämtliche damit verbundene "Master-Theorien« oder -Narrative, Vereinheitlichungen oder Vereinfachungen. Stattdessen fordern sie genaue historische, geographische und sozio-kulturelle Verortungen der sozialen Praxen und politischen Kämpfe von Frauen. "Scattered Hegemonies«, so der Titel ihres Sammelbandes, die sich an unterschiedlichen Orten und auf verschiedenen Ebenen des politischen und sozialen Handelns jeweils spezifisch aus makro-ökonomischen Strukturen, patriarchalen Nationalismen, vermeintlich rauthentischen Traditionalismen, lokalen Machtstrukturen und rechtlich formalisierten Unterdrückungsmustern zusammensetzen (Grewal / Kaplan 1994, 17), bilden für sie die Referenzpunkte transnationaler feministischer Praxen und Analysen. Transnational feministisch bedeutet in dieser Sichtweise also ganz explizit keine fixierbaren politischen Standpunkte oder politische Handlungsräume, sondern vielmehr die Summe einer Vielzahl modernitätskritisch-postmoderner, grenzüberschreitender feministischer Perspektiven des Denkens und Handelns.

Demgegenüber setzen Alexander und Mohanty in ihrem Buch mit dem Titel "Feminist Genealogies, Colonial Legacies, Democratic Futures" den Schwerpunkt ihres Arguments auf die politischen Notwendigkeiten und Möglichkeiten transnationaler feministischer Solidaritäten. Der Text geht 
zurück auf eine Kooperation von women of colour verschiedener Nationalitäten aus dem Jahr 1988, deren Ziel darin bestand, durch genaue Historisierungen die "whiteness" des internationalen Feminismus und der liberalen Frauen- und Geschlechterstudien (in den USA) offenzulegen und zugleich eine Plattform des Austausches über radikale feministische Gerechtigkeitskonzeptionen zu bieten. Diese Perspektive ist prägend für Alexander und Mohantys feministisch-materialistische Kritik an den Verhältnissen von Staat und Kapital, die "race erstens als konstitutiv für die Herausbildung internationaler Kapitalverhältnisse hervorhebt und zweitens als bestimmend für die Wechselverhältnisse zwischen den konkreten, lokalen Erfahrungen und Praxen von Frauen weltweit und den strukturellen Bedingungen, in die sie eingebettet sind, in die Analyse hineinnimmt. Den politischen Horizont des Ansatzes von Alexander/Mohanty bildet ein normatives Konzept von "feminist democracy «, das als antikolonial, antikapitalistisch, basisbezogen-kollektivistisch und transnational definiert wird (Alexander/Mohanty 1997, 27ff.). Transnationaler Feminismus ist in dieser Perspektive ein dekoloniales Projekt der Solidarität, das die Kategorien der Intersektionalität historisch und (geo)politisch zueinander ins Verhältnis setzt.

Eine grundsätzlich andere Sicht auf transnationale Feminismen entwerfen Beiträge aus der Genderforschung über Internationale Politik, die seit dem Ende der 1990er Jahre danach fragen, wie transnationale feministische Netzwerke die reale Politik der internationalen Staaten verändern. Entgegen den jahrzehntealten Annahmen des Mainstreams in den internationalen Beziehungen, wonach internationale Politik als eine nahezu ausschließliche Angelegenheit von Nationalstaaten und ihren internationalen Institutionen erschien, kennzeichnen diese Forschungen die transnationalen Netze aus Frauenbewegungen, NGOs und hochspezialisierten Gender-Expert $\star_{i n n e n}$ als wichtige Quelle der Veränderung internationaler Normen. Margaret E. Keck und Kathryn Sikkink (1998) waren mit ihrer Studie "Activist beyond borders", die u.a. die erfolgreiche Politik der transnationalen FrauenMenschenrechte-Netze gegen Gewalt gegen Frauen untersucht, unter den Ersten in diesem Feld, die untersuchten, auf welche Weise transnationale Bewegungspolitiken Veränderungen in der internationalen Politik erreichen. Zugleich arbeiteten sie empirisch heraus, wie transnationale Bündnisse in umgekehrter Politikrichtung progressive internationale Normen nutzen, um Nationalstaaten zur Implementierung von mehr Geschlechtergleichheit zu bewegen. Transnational ist in dieser Sichtweise daher ein analytischer Begriff für eine verschiedene politische Handlungsebenen überspannende bzw. verbindende Zivilgesellschaftspo- 
litik, die prinzipiell in beide Richtungen funktionieren kann: von lokal über national ins Internationale oder umgekehrt aus dem Internationalen über die nationale Ebene bis ins Lokale.

Zwischen diesen drei Polen bzw. innerhalb des diskursiven Dreiecks, das sie markieren, und zugleich in vielfältigen Wechselverhältnissen mit den Entwicklungen, Brüchen und Krisen der Weltpolitik, entspannen sich in den letzten 25 Jahren vielfältige, zum Teil auch widersprüchliche Theorien und Praxen transnationaler Feminismen. Dabei haben die verschiedenen Stränge des Diskurses immer voneinander gelernt, sich ergänzt und flankiert und zum Teil vehement miteinander gestritten. So entstanden und entstehen auch weiterhin vielschichtige Dynamiken nach außen wie nach innen.

\section{Zukunftsansichten}

Die Beiträge zu diesem Heft reflektieren die Spannbreite der verschiedenen erkenntnistheoretischen und politischen Ansätze transnationaler Feminismen ebenso wie verschiedene Formen der Synthese von Konzepten und Positionen. Für eine zukunftsorientierte Diskussion transnationaler Feminismen halten wir insbesondere vier Aspekte für wesentlich, die auf unterschiedliche Weise von allen hier versammelten Texten diskutiert werden. Erstens fragen alle Autorinnen nach Bewegungspolitiken oder nach Praxen von Aktivist $\star_{i n n e n}$ und ihren gegenhegemonialen Projekten und Diskursen, die nach wie vor im Zentrum transnationaler Feminismen stehen. Zweitens stellt der Ansatz der Intersektionalität, immer verknüpft mit den Fragen nach ihren politischen Horizonten in transnationalen Entwicklungen, einen Knotenpunkt der Beiträge dar. Drittens diskutieren alle Texte räumliche Bezüge des Transnationalen, indem sie Handlungsebenen und Handlungsräume von Aktivismus als entscheidende Dimension der analytischen Verortung sozialer Praxen begreifen. In allen Texten geht es viertens um die Suche nach Möglichkeiten transnationaler Solidaritäten, die immer schon die Geschichte transnationaler Feminismen bewegt hat. Solidarität ist darin freilich nicht einfach ein politischer Selbstzweck, sondern sowohl Grundlage als auch Mittel der angestrebten emanzipativen Transformationen und Dekolonisierungen.

Dagegen bilden die hier vertretenen thematischen Zugriffe auf transnationale feministische Praxen zwangsläufig einen kleinen Ausschnitt der möglichen Annäherungen an das Feld ab. Nur sehr selektiv können die Texte auf Entwicklungen in verschiedenen Regionen der Welt und in 
verschiedenen Feldern feministischer Politiken verweisen, andere Prozesse und Zusammenhänge, wie etwa, um nur einen Kontext herauszugreifen, Migration und Grenzregime, bleiben völlig unthematisiert. Eine gewisse Konzentration der Beiträge auf afrikanische Perspektiven ist sicherlich eigenen Arbeitsschwerpunkten geschuldet, spiegelt aber auch jüngere Dynamiken der Transregionalisierung von Weltpolitik wider, in denen afrikanische Regierungen sowie afrikanische Erfahrungen und Perspektiven vor allem im Zusammenhang der Intensivierung asiatisch-afrikanischer Interaktionen eine wichtige Rolle spielen. Zugleich war und ist der panafrikanische Feminismus für die Politisierung der Verhältnisse von "race u und Gender und die gesamten transnationalen feministischen Diskurse darum besonders wichtig.

Das Heft beginnt mit einem Email-Gespräch über transformatorische Potenziale transnationaler Feminismen, das den Blick sowohl zurück in die Geschichte als auch nach vorne in die Zukunft richtet und vier Kolleginnen befragt, die mit jeweils unterschiedlichen Erfahrungen und theoretischen Verortungen in die Diskurse um transnationale Feminismen involviert sind. Christa Wichterich, Nikita Dhawan, Esther Franke und Radwa Khaled erörtern Perspektiven, Praxen und Wandlungsprozesse transnationaler Feminismen und Bewegungspolitiken in ihren Wechselbeziehungen zu den sich verändernden Bedingungen internationaler Politik. Vor dem Hintergrund der derzeitigen Krisen der Weltpolitik diskutieren sie gegenhegemoniale feministische Entwürfe und mögliche Beiträge aus dem Globalen Norden zu den Praxen transnationaler feministischer Solidarität.

Der zweite Beitrag von Carole Boyce Davies über die historische Verwobenheit der Freiheitskämpfe panafrikanischer und Schwarzer Us-amerikanischer Feminist $\star_{i n n e n}$ belegt eindrücklich, dass Intersektionen von "race und Gender theoretisch wie politisch schon seit mehr als hundert Jahren Gegenstand transnationaler feministischer Kämpfe sind. An der empirisch dicht rekonstruierten Geschichte des feministischen Panafrikanismus zeigt Boyce Davies, wie "race und Gender als sich wechselseitig konstituierende Kategorien in den feministischen Kämpfen um Gleichheit und Freiheit zwischen dem afrikanischen Kontinent und den USA verhandelt wurden und zu verschiedenen Zeiten in verschiedenen politischen Kontexten der Geschichte unterschiedliche Bedeutungen trugen. Deutlich wird damit auch die große Wichtigkeit der Archive feministischen Wissens für die Theoriebildung, die in westlichen (weißen) Kontexten bisher allerdings kaum zur Kenntnis genommen werden. 
Im Text von Johanna Leinius über die Translokalisierung lateinamerikanischer Feminismen geht es ebenfalls um sich gegenseitig bedingende Kämpfe und die Frage nach den lokalen Verortungen von feministischen Praxen und Erfahrungen. Leinius diskutiert auf der Grundlage eigener empirischer Beobachtungen der lateinamerikanischen feministischen Treffen ("encuentros«), wie Solidarität auf dem Kontinent unter den Bedingungen der Bandbreite an zum Teil scharf gegensätzlichen Lebensverhältnissen und Erfahrungen ausgehandelt werden kann. Verortungen, Kontextualisierungen und Intersektionalität sind für Leinius dabei die Schlüssel, um zu verstehen, wie lateinamerikanische Feminist $\star_{i n n e n}$ dialog- und verhandlungsfähig bleiben können. Daher setzt Leinius dem politischen Verständnis von Transnationalität aus den Internationalen Beziehungen die Methode und Perspektive der Translokalisierung entgegen. Ähnlich dem poststrukturalistischen Ansatz von Grewal/Kaplan betont Translokalisierung die Prozesshaftigkeit politischer Aushandlungen und die Notwendigkeit der tiefenscharfen Verortung sozialer Praxen und politischer Perspektiven. Transnationale respektive translokale Sphären des politischen Handelns sind demnach keine a priori definierten Räume, sondern bilden sich in den Prozessen der Auseinandersetzung. Wie der Text von Boyce Davies verweist auch Leinius' Beitrag nicht zuletzt darauf, dass Intersektionalität als zentrale Dimension der Kämpfe und Praxen transnationaler Frauenbewegungen der akademischen Begriffsbildung deutlich vorgelagert ist.

Auch Ina Kerners Beitrag dreht sich um Intersektionalität und transnationale Solidaritäten - allerdings in einer umgekehrten Perspektive, die Spannungsverhältnissen zwischen beiden Konzepten politiktheoretisch nachspürt. Ausgehend von den Erfahrungen feministischer Aktivist $\star_{i n-}$ nen in Pakistan, für die poststrukturalistische bzw. postkoloniale Ansätze von Intersektionalität mit Verlusten an globaler Solidarität und politischer Durchsetzungskraft verbunden sind, fragt Kerner danach, welche grenzüberschreitenden Solidaritäten in einer Welt widersprüchlicher globaler Interdependenzen und Asymmetrien von lokal bis international überhaupt möglich sind. Vermeintlich respektvolle, weil die Fallen des Neokolonialismus umgehende Rückzüge westlicher Feminisit $\star_{\text {innen }}$ aus gemeinsamen Süd-Nord-Kämpfen gegen kulturell-religiös verbrämte Verletzungen von FrauenMenschenrechten kritisiert Kerner als "feministischen Provinzialismus". Ein dahinterstehendes, identitätspolitisch aufgeladenes Verständnis von Intersektionalität kennzeichnet sie als »semi-intersektional«, weil es die transnational-räumlichen Dimensionen von Herrschaftsverhältnissen nicht ausreichend bedenkt. Gegen eine theoretische Beschrän- 
kung auf die Dezentrierung und Kontextualisierung von Kategorien und Erfahrungen plädiert Kerner dafür, kulturelle und religiöse Partikularitäten immer auch in ihren historischen und geopolitischen Verwobenheiten zu analysieren. Erkennbar wird in dieser Argumentation die oben diskutierte Grundspannung zwischen den verschiedenen theoretischen Fundamenten und Praxen transnationaler Feminismen. $\mathrm{Ob}$ und wie sich poststrukturalistisch-postkolonial fundierte Ansätze der Dezentrierung von Wissens- und Machtverhältnissen und materialistisch- feministische Kritikperspektiven, deren Horizont letztlich immer die politische Veränderung von Macht- und Herrschaftsverhältnissen bildet, tatsächlich miteinander verbinden lassen, ist sicherlich eine der zentralen Zukunftsfragen transnationaler Feminismen. Und zweifellos werden diese Widersprüche noch für geraume Zeit ein Movens innerfeministischer Transformationen sein.

Einen ganz anderen Aspekt grundlegender Veränderungen im transnationalen Feminismus bespricht der Beitrag von Rirhandu Mageza-Barthel, der nach den Bedeutungen von feministischem Aktivismus in den aktuellen Prozessen der Multipolarisierung und Transregionalisierung der Weltpolitik fragt. An der Geschichte der feministischen Beziehungen zwischen China und Südafrika verdeutlicht Mageza-Barthel deren komplex verwobenes Eingebundensein in die teilweise widersprüchlichen Prozesse nationaler, regionaler und transnationaler Politik. Während Kooperationen rund um die Peking-Konferenz, in deren Kontext sich die beschriebenen feministischen Transregionalisierungsprozesse deutlich konturierten, noch "post-bi-polar" geprägt sind, haben sich im Rahmen der jüngeren Süd-Süd-Beziehungen mit dem ökonomischen Aufstieg von China, Brasilien, Indien und Südafrika ganz neue Dynamiken entfaltet. Obwohl damit auch neue Gelegenheitschancen für feministische Kooperationen entstehen, halten sich bestimmte Gruppen südafrikanischer Aktivisit ${ }^{\star}$ innen aus diesen neuen Handlungsräumen weitgehend heraus und ziehen die Kontinuitäten transnationaler feministischer Kooperationen den unklaren Perspektiven transregionaler Neustrukturierung deutlich vor. Inwiefern diese Haltung auch auf den historischen Erfahrungen südafrikanischer Feminist $\star_{i n n e n}$ mit den wechselhaften Phasen der Zusammenarbeit zwischen dem ANC und der kommunistischen Partei Chinas beruht, arbeitet der Beitrag an verschiedenen Facetten der historischen Prozesse von Transregionalisierung heraus.

Einer anderen Version von Transregionalismus widmet sich der Beitrag von Amina Mama in der Rubrik »In der Diskussion", der die Geschichte des panafrikanischen feministischen Zeitschriftenprojektes feminist africa 
rekonstruiert. In seiner ursprünglichen Fassung leitete der Artikel das im Jahr 2017 erschienene, vorerst letzte Heft dieser (nicht nur) für den panafrikanischen Feminismus so bedeutungsvollen open access Zeitschrift ein. Die politische Kraft feministischen Schreibens und der Zusammengehörigkeit von Theoriebildung und Aktivismus, die befreiende Wirkung von Bewusstseinsbildung und nicht zuletzt die Stärke von Kooperationen und Solidaritäten zwischen Feminist ${ }^{\star}$ innen aus Wissenschaft, Kunst, Politik und (Basis)Aktivismus des gesamten Kontinents sind die Themen, die Mama in ihrer Rückschau diskutiert. Dabei bilden die Kolonialität von Wissensverhältnissen und die Persistenz der westlichen Dominanz in der Wissensproduktion die gleichsam sandere transnationale Seite, die dieses Wissensprojekt stets (mit)angetrieben hat. Fast zwanzig Jahre lang hat feminist africa feministische Forschungen und Beiträge aus unterschiedlichsten politischen, ökonomischen und kulturellen Kontexten des Kontinents zusammengefasst zu einem (gegen) öffentlichen feministischen Raum, der weit mehr als nur Hochschulpolitik und wissenschaftliche Diskurse auf dem afrikanischen Kontinent beeinflusst hat. Der "Kraft panafrikanischen feministischen Denkens", wie Amina Mama ihren Text überschrieben hat, in genau dieser Ausgabe der feministischen studien gebührenden Raum zu geben, war uns als Herausgeberinnen gemeinsam mit dem Redaktionsteam ein besonderes Anliegen.

Das Interview mit Awin Swed in der Rubrik »Im Gespräch" über die kurdische Frauenbewegung in Nordsyrien, dessen besondere aktuelle Bedeutung zu dem Zeitpunkt, als es geführt wurde, noch gar nicht klar war, vergegenwärtigt noch einmal nachdrücklich und auf drastische Weise, wie sehr die Perspektiven feministischer Kämpfe mit den Konfigurationen inter- und transnationaler Politik verbunden sind - und damit auch, welche Bedeutung den konkreten Orten des Transnationalen für den Gesamtzusammenhang zukommt. Wie kein anderer derzeitiger Konflikt ist der Syrienkonflikt durch die Beteiligung einer Vielzahl von Staaten, die um die Neuordnung der Region kämpfen, ein Austragungsort der Auseinandersetzungen um die Neuformierung globaler Politik. Das Interview macht begreiflich, wie die kurdische (Frauen-)Bewegung durch ihre lokalen Praktiken und Politiken inmitten dieses Konflikts eine alternative Zukunft entwirft und Mechanismen und Politikstrategien entwickelt, um Gleichheit und Gerechtigkeit basisdemokratisch umzusetzen. Dabei sind Diskurse und Praxen der transnationalen Solidarität von Beginn an integraler Bestandteil und langfristige Perspektive dieses politischen Projekts gewesen. 
Revolutionäre Umbrüche, Gegenentwürfe, Handlungsmacht und alternative Formen der Repräsentation von Frauen sind die Stichworte, die für die Graffitis in der Rubrik Bilder und Zeichen stehen. Sie stammen aus Ägypten und stehen im Kontext der revolutionären Zeiten 2011/2012, in denen Frauen sich außerordentlich stark politisierten und engagierten. Zugleich verweisen sie auf die vielfältigen Gewalterfahrungen, mit denen Frauen in diesen Zeiten konfrontiert wurden. Weiblich konnotierte Körper und Sexualität wurden (auch) in den gesellschaftlichen Kämpfen in Ägypten zu zentralen Austragungsorten der Auseinandersetzung um die zukünftige gesellschaftliche und politische Ordnung. Die Symbolkraft der Abbildungen für transnationale feministische Kämpfe und die Anforderungen an transnationale feministische Solidaritäten ist in mehrfacher Hinsicht beeindruckend und sollte den stransnationalen Archiven feministischen Wissens unbedingt erhalten bleiben.

\section{Epilog}

Dass eine Zukunftsbilanz zwangsläufig ein offener Wechsel auf bzw. in die Zukunft bleiben muss, war uns als Herausgeberinnen bei der Heftplanung bewusst. Unser Ziel war es, Perspektiven transnationaler Feminismen in die Zukunft zu dokumentieren und einen Vorschlag zu entwerfen, sie vorläufig zu rahmen. Dessen konzeptionelle Aspekte sind zu größeren Teilen verbunden mit Arbeiten von Uta Ruppert, die sich seit der Pekingkonferenz mit Theorie und Praxis transnationaler Feminismen befasst hat und die daher diese Einleitung verantwortet. Sämtliche anderen Aspekte der Herausgabe dieses Heftes sind eine Arbeit des Dreierteams Uta Ruppert, Tanja Scheiterbauer und Katharina Liebsch, die an dieser Stelle die Gelegenheit nutzen möchten, allen Autor`innen sowie dem gesamten Redaktionsteam der feministischen Studien für die sehr gute Zusammenarbeit und ganz besonders Regine Othmer für die ebenso aufwendige wie umsichtige, aber oft unsichtbare Arbeit der Endredaktion herzlichst zu danken. 


\section{Literatur}

Alexander, M. Jacqui/Mohanty, Chandra Talpade (1997): Introduction: Genealogies, Legacies, Movements. In: Alexander, M. Jacqui / Mohanty, Chandra Talpade (Hrsg.): Feminist Genealogies, Colonial Legacies, Democratic Futures. New York, London, xiii-xlii.

Antrobus, Peggy (2004): The Global Women's Movement. Origins, Issues and Strategies. London.

Baksh, Rawwida / Harcourt, Wendy (Hrsg.) (2015): The Oxford Handbook of Transnational Feminist Movements. New York.

Braig, Marianne / Wölte, Sonja (2003): Common Ground or Mutual Exclusion? Women's Movements and International Relations. London.

Caglar, Gülay / Prügl, Elisabeth / Zwingel, Susanne (Hrsg.) (2013): Feminist Strategies in International Governance. New York.

Combahee River Collective (1978): A Black Feminist Statement. In: Eisenstein, Zillah (Hrsg.): Capitalist Patriarchy and the Case for Socialist Feminism. New York: Monthly Review Press, 362-372.

Correa, Sonia / Reichmann, Rebecca (1994): Population and Reproductive Rights: Feminist Perspectives from the South. London, New Jersey.

Desai, Manisha (2015): Critical Cartographies, Theories and Praxis of Transnational Feminisms. In: Baksh, Rawwida/Harcourt, Wendy (Hrsg.): The Oxford Handbook of Transnational Feminist Movements. New York, 116-130.

Grewal, Inderpal/Kaplan, Caren (1994): Introduction: Transnational Feminist Practices and Questions of Postmodernity. In: Grewal, Inderpal/Kaplan, Caren (Hrsg.): Scattered Hegemonies. Postmodernity and Feminist Practices. Minneapolis, London, 1-33.

Holland-Cunz, Barbara / Ruppert, Uta (Hrsg.) (2000): Frauenpolitische Chancen globaler Politik. Verhandlungserfahrungen im internationalen Kontext. Wiesbaden.

Kapur, Ratna (2002): The Tragedy of Victimization Rhetoric: Resurrecting the "Native» Subject in International / Post-Colonial Feminist Legal Politics. In: Harvard Human Rights Journal. 15 (1), 1-38.

Keck, Margaret E./Sikkink, Kathryn (1998): Activists beyond Borders: Advocacy Networks in International Politics. Ithaca.

Lenz, Ilse/Schwenken, Helen (2001): Themenschwerpunkt: Lokal, national, global? Frauenbewegungen, Geschlechterpolitik und Globalisierung. In: Zeitschrift für Frauenforschung \& Geschlechterstudien. (1+2).

Lutz, Helma / Amelina, Anna (2017): Gender, Migration, Transnationalisierung. Eine intersektionelle Einführung. Bielefeld.

McLaren, Margaret A. (2017): Decolonizing Rights: Transnational Feminism and »Women's Rights as Human Rights«. In: McLaren, Margaret A. (Hrsg.): Decolonizing Feminism. Transnational Feminism and Globalization. Lanham, 205-286.

Meyer, Mary E. / Prügl, Elisabeth (Hrsg.) (1999): Gender Politics in Global Governance. Oxford.

Moghadam, Valentine M. (2005): Globalizing Women. Transnational Feminist Networks. Baltimore.

Ruppert, Uta (Hrsg.) (1998): Lokal bewegen - global Verhandeln. Internationale Politik und Geschlecht. Frankfurt am Main. 
Ruppert, Uta (2001): Von Frauenbewegungen zu Frauenorganisationen, von Empowerment zu FrauenMenschenrechten: Über das Globalwerden internationaler Frauenbewegungspolitik. In: Österreichische Zeitschrift für Politikwissenschaft. 30 (2), 203-219.

Ruppert, Uta (2015): Menschenrechte als ambivalentes Instrument globaler Politik: das Beispiel Frauenpolitik. In: Reder, Michael/Cojocaru, Mara-Daria (Hrsg.) Zur Praxis der Menschenrechte. Formen, Potenziale und Widersprüche. Stuttgart, 104-115.

Schild, Verónica (2014): Die Spezifik lateinamerikanischer Feminismen im Kontext neoliberaler Regulierung. Beunruhigende Divergenzen - Produktive Ambivalenzen. In: Das Argument. 308 (3), 356-368.

Sen, Gita/Durano, Marina (2014): The Remaking of Social Contracts: Feminists in a Fierce New World. London.

Sen, Gita/Grown, Caren (1987): Development, Crisis and Alternative Visions. Third World Women's Perspectives. New York.

UN Women (2016): UN Women Annual Report 2015-2016. New York: UN Women.

United Nations (2015): Convention on the Elimination of All Forms of Discrimination against Women. Consideration of reports submitted by States parties under article 18 of the Convention. Seventh and eighth periodic reports of States parties due in 2014. Germany. ( Nr. CEDAW/C/DEU/7-8) United Nations.

Uremović, Olga / Oerter, Gundula (Hrsg.) (1994): Frauen zwischen Grenzen. Rassismus und Nationalismus in der feministischen Diskussion. Frankfurt am Main, New York.

Wichterich, Christa (1995): Frauen der Welt. Vom Fortschritt der Ungleichheit. Göttingen.

Zwingel, Susanne (2012): How Do Norms Travel? Theorizing International Women's Rights in Transnational Perspective. In: International Studies Quarterly. 56 (1), 115129. 